\title{
ICOSAHEDRAL SUPERSYMMETRY
}

\author{
J.A.de Wet \\ Box 514, Plettenberg Bay, 6600, \\ South Africa
}

Keywords: $E_{6}, E_{8}$, Weyl Group,Mass from Entropy,Supersymmetry.

\begin{abstract}
The Exceptional Lie Algebra $E_{6}$ used by the Author as a basis for the Standard Model of the Elementary Particles is a subalgebra of the Lie algebra $E_{8}$ which in turn is the Lie algebra of the icosahedral group by the McKay correspondence.It is possible to introduce a mass proportional to an entropy given by the the number of permutations of the elements of $E_{6}, E_{8}$ labeled by the Weyl group W.In this way the masses of the top-quark pair uu and electron are derived without any appeal to QCD and a mass of approximately $19 \mathrm{TeV}$ is predicted for supersymmetric particles.
\end{abstract}

\section{Introduction}

This contribution is an addendum of the work 'Icosahedral Symmetry and the MSSM'[6]. The icosahedron has 20 faces and 12 vertices and is a Pla- tonic solid that can be rotated into itself about 6 diagonals. Constituting the Icosahedral Group that has the Lie algebra $\mathrm{E}_{8}$ by the McKay Correspon- dence. The Exceptional Lie group $\mathrm{E}_{6}$ is a subalgebra of $\mathrm{E}_{8}$ that has been used by de Wet [8] as a basis for the Standard Model of the elementary particles who employed the E6 triality subalgebra $\left(\mathrm{su}_{3}\right)_{\text {color }} \times\left(\mathrm{su}_{3}\right)_{\text {spin }} \times\left(\mathrm{su}_{3}\right)_{\text {isospin }}$ which also defines the 27 lines on a Cubic surface shown in Fig.1 according to Coxeter ([4] Section 12.3). According to Hunt ([10],Ch.4)these lines correspond to the 27 fundamental weights of of $E_{6}$. Fifteen of these lines are a double 6 which may be labeled by the 3 sets of commuting operators (called Synthemes by Hunt)in equations 2,3,4. The remaining 6 synthemes

$$
(14,25,03),(14,35,05),(24,15,03),(24,35,01),(34,15,02),(34,25,01)
$$

will be shown to carry no charge.

The pair ab of 2 numbers in equations 1-4 is shorthand for a $4 \times 4$ ma- trix Eab employed by Eddington that is isomorphic to a Dirac matrix $\gamma_{v}$ as described by de Wet[7] but has a transparent physical interpretation as will appear below. The 3 sets of commuting operators are as follows. The first set is

$$
(12,34,05),(31,24,05),(23,14,05)
$$

which represents nucleons.Here $\mathrm{E}_{12}, \mathrm{E}_{23}, \mathrm{E}_{31}$ are spin rotations about $\mathrm{x}_{1}, \mathrm{x}_{3}, \mathrm{x}_{2}$ accompanied by parities $\mathrm{E}_{34}, \mathrm{E}_{24}, \mathrm{E}_{14}$ that can reflect a spin . $\mathrm{E}_{05}$ is a coor- dinate in isospace. The second set is

$$
(12,35,04),(31,25,04),(23,15,04)
$$

where there are again 3 spin axes and a coordinate of isospace but no parity in a 5 -space so we interpret these synthemes as possibly mapping super- symmetric particles. The coordinates $(15,25,35)$ would be those of a Del Pezzo surface for $\mathrm{E}_{8}$ as described by Manivel [11] which will be seen in the next Section to carry a mass of approximately $19 \mathrm{eV}$. The third set

$$
(12,03,45),(31,02,45),(23,01,45)
$$

now describes particles with the third component isospin coordinate but with no parity and coordinates $(01,02,03)$ that as yet has no interpretation. 
Another set of 15 synthemes is considered in [9] on a Knot Theory which describes Fig, 1 as a toric knot that could lie in a knotted space which is believed to carry the vortex lines of classical and quantum fields that arise naturally as excitations which carry helicity. In this contribution the inner ring of Fig. 1 in the 6-d projective space $\mathrm{CP}^{3}$ is supposed to compactify to a point at the origin for the $\mathrm{e}^{ \pm}, \mathrm{e}_{v}$ triality, when quarks in the 6-space contract to nucleons in a $3 \mathrm{~d}$ projective space $\mathrm{P}^{3}$ which holds 15 synthemes according to Barth and Nieto [2].Twelve synthemes remain on the outer surface of Fig. 1 contributed by 6 for the protons and neutrons of equation (3) and another 6 for their antiparticles. The remaining 3 for the electrons and electron neutrino are labeled by

$$
(011,022, \mathrm{O} 33),(110,220,330)(101,202,303)
$$

In this process the masses $\mathrm{m}_{\tau}, \mathrm{m}_{v}$ for the $\tau$ and $\mu$ mesons reappear as stable deuterium according to the relationship

$$
\mathrm{m}_{\tau}+\mathrm{m}_{v}=\mathrm{m}_{\mathrm{p}}+\mathrm{m}_{\mathrm{n}}+\mathrm{m}_{\mathrm{e}}
$$

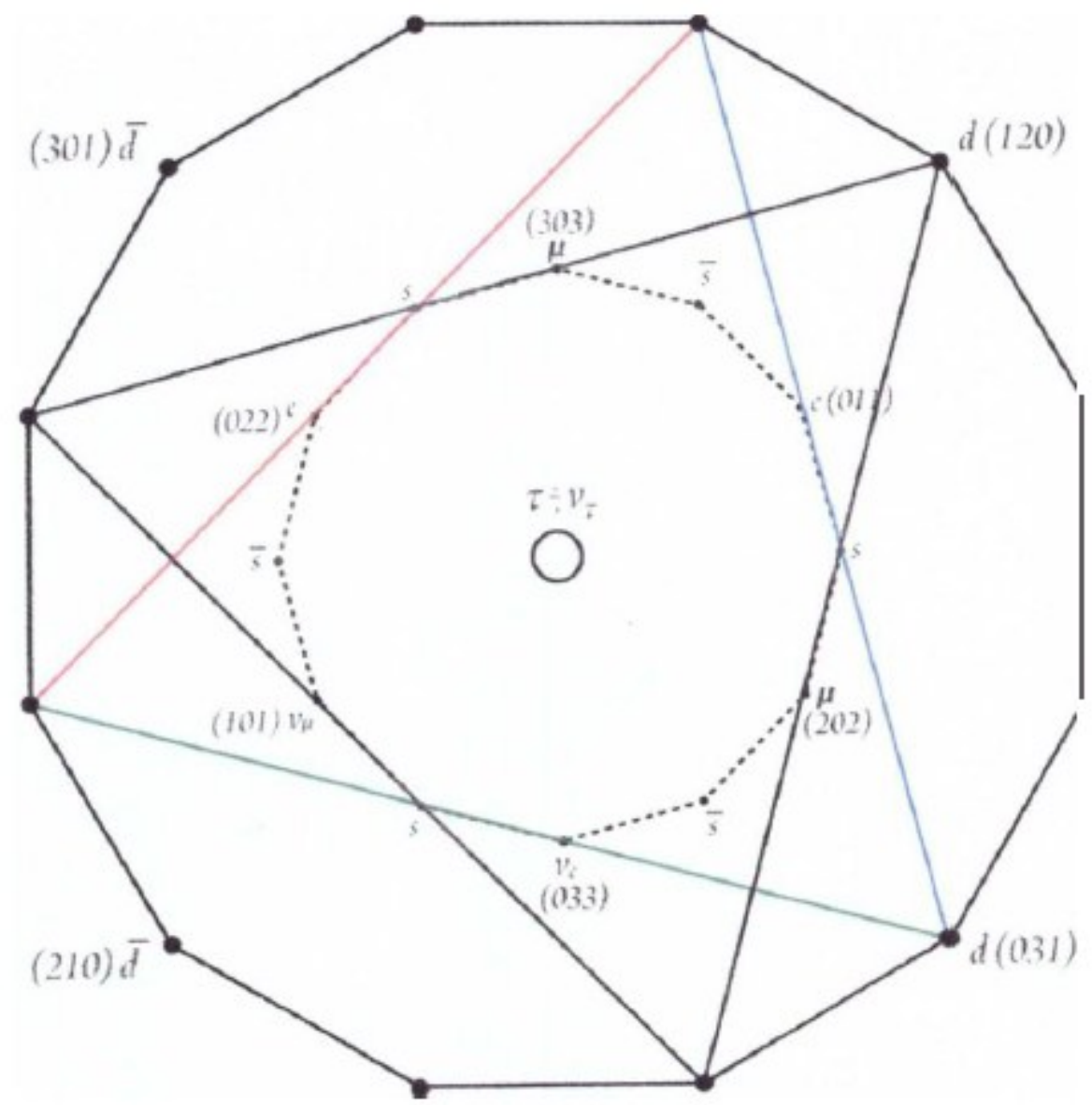

Fig.1

Figure 1 taken from Coxeter [3,4] shows an arrangement of the 27 lines governed by $\left(\mathrm{su}_{3}\right)^{3}$. Here Coxeters labeling $(0 \mu v, v 0 \mu, \mu v 0)$ is employed for lines on a tritangent to the cubic surface that can be rotated into itself by $\omega=120$ degrees. Another labeling of the 27 particles in the Standard Model is due to the Author based on work by Slansky [12]. In this way the outer ring carries the proton and neutron tritangents whose vertices are 3 quarks. Two more tritangents which carry the antiparticles have not been drawn . Unfortunately because of a hitch in transferring to Word, two of the vertices of the colored proton tritangent have been lost, namely (working anti-clockwise from the top) $\mathrm{u}(012), \mathrm{u}(021)$. Similarly $\mathrm{d}(230)$ and $\mathrm{u}(310)$ are missing from the black neutron tritangent. 


\section{Supersymmetry and the Origin of Mass}

A new approach to the origin of mass appears in Ref.[7] where mass is related to the entropy of organisation. Specifically to the number of permutations

encountered in a subalgebra $\left(\mathrm{su}_{3}\right) \times\left(\mathrm{su}_{3}\right) \times\left(\mathrm{su}_{3}\right)$ or $\left(\mathrm{su}_{3}\right)^{3}$ of $\mathrm{E}_{6}$ which are the Weyl group $\mathrm{W}$ of the elements in the representation. For example there are $3 !=6$ ways in which an up-quark u,represented by su3 can combine with another up-quark from $\mathrm{su}_{3}$ and $6^{2}$ in which uu can join a down-quark to yield a proton.So the number of possible permutations is $\left|\mathrm{W}\left(3 \mathrm{~A}_{2}\right)\right|=36$ as given by Manivel ([11],p9)and believed to be a measure of the uu mass

$$
\mathrm{m}_{\mathrm{uu}}=\eta \times 216 \approx 6 \mathrm{MeV}
$$

where $\eta=27.7 \mathrm{keV}$ is a constant of proportionality.

Then in the case of the electron we may use the alternative representation $3\left|\mathrm{~W}\left(\mathrm{~A}_{2}\right)\right|=18$ of $\left(\mathrm{su}_{3}\right)^{3}$ in $\mathrm{E}_{6}$ employed by Adams [1] that yields a mass

$$
\mathrm{m}_{\mathrm{e}}=27.7 \times 18=498.6 \mathrm{keV}
$$

which is close to the recorded value when the mass of the electron neutrino is added.

This concludes the masses of the stable protons and electrons, but we can end with a subalgebra of $E_{8} \supset E_{6}$. The number of possible permutations of $E_{8}$ is 696729600 according to Adams [1] Ch.10. Therefore if we take $\mathrm{W}\left(\mathrm{E}_{8}\right)$ to be the Weyl group representing supersymmetry the number of supersymmetric states would be 696729600 corresponding to a mass of

$696729600 \times 27.7 \approx 19 \mathrm{Tev}$

if there are no subalgebras. But such a mass has not been achieved at the LHC at CERN.

\section{References}

[1] J.F.Adams, Lectures on Exceptional Lie Groups, The University of Chicago Press, 1996.

[2] W.Barth and I.Nieto, Abelian surfaces of type (1,3) and quartic surfaces with 16 skew lines, J.Algebraic Geometry 3 (1994) 173-222.

[3] H,S.M.Coxeter, The polytope 221 where 27 vertices corespond to the lines on the general cubic surface, American J. of Mathematics, 62 (1940) 457-486.

[4] H.S.M.Coxeter, Regular Complex Polytopes,2nd ed.,Cambridge Univer- sity Press, 1991.

[5] J.A.de Wet, A group theoretical approach to the many nucleon problem, Proc.Camb.Phil.Soc.,70 (1971)485-496.

[6] J.A,de Wet, Icosahedral symmetry in the MSSM, Int.Mathematical Forum,3(2008)777282,(Available on-line at Hikari Ltd).

[7] J.A.de Wet, A Standard Model algebra, Int.Mathematical Forum,7(2012)49-52.

[8] J.A.de Wet, On the strong force without QCD, Int.Mathematical Forum,7(2012)129-132.

[9] J.A.de Wet, Particle knots in Toric Modular Space, Bulletin of Society for Mathematical Services@ Standards,3(2014)54-59.

[10] Bruce Hunt, The Geometry of some Arithmetic Quotients, Lecture Notes in Mathematics ,1637, Springer(1996).

[11] L.Manivel, Configuration of lines and models of Lie algebras, arXiv;math.AG/0507118.

[12] R.Slansky, Group Theory for Unified Model Building, Unity of Forces in Universe, Ed.A.Lee, World Scientific, 1992. 